\title{
Droplet Impact Simulation of Hydrophobic Patterned Surfaces by Computed Fluid Dynamics
}

\author{
Zhiru Yang*, Chongchong Zhu and Nan Zheng \\ School of Mechanical Engineering,Jiangsu University, Zhenjiang 212013, China \\ *Corresponding author e-mail: wan_009@126.com
}

\begin{abstract}
Droplet Impact Simulation is a vital way to investigate the liquid and superhydrophobic surface properties of materials. In this examination, the droplet are investigated chiefly dependent on the Cassie state and the Wenzel state. The VOF method is utilized for numerical reproduction to research the instrument of the droplet's effect on the finished surface and to present a basic and viable strategy to figure Contact edge to indicate surface hydrophobicity. The reenactment results demonstrate that the surface hydrophobicity can be adequately enhanced by planning distinctive smaller scale surface on the hydrophilic surface. With the expansion of speed, the consistent state contact edge of the finished surface is clearly diminished. What's more, by quantitatively examining the development of droplets on the miniaturized scale finished surface, it is discovered that the consistent state contact edge is identified with the base spreading width of the main droplet after effect, and the littler the distance across, the bigger the contact point.
\end{abstract}

KEYWORDS: patterned surface, impact simulation, steady-state contact angle, hydrophobic, patterned surfaces

\section{Introduction}

Due to its excellent water-repellency, superhydrophobic surfaces have attracted a great deal of attention. Especially, the surface-to-surface transition from hydrophilic to hydrophobic properties has triggered a continuous and intensive study [1, 2]. For smooth and uniform material surfaces, the maximum contact angle that can be achieved without any special treatment is less than $120^{\circ}$ [3]. Superhydrophobic surfaces are more likely to droplet droplets from the surface or rebound upon impact on the surface. Like the superhydrophobic lotus leaf surface, rain droplets can easily roll off the surface in a spherical shape [4]. In nature, in addition to lotus leaf surface, there are also some plant and animal surfaces have superhydrophobic properties. The results show that the micro-scale, nano-scale or micro-nano composite patterned can significantly improve the quality of surface water repellent properties $[5,6]$. In 
Academic Journal of Engineering and Technology Science

ISSN 2616-5767 Vol. 1, Issue 1: 49-55, DOI: 10.25236/AJETS.020006

many practical applications, droplets always undergo a rapid movement, such as high-speed spraying and rapid impact on the surface or rapid sliding on the surface [7]. In these processes, droplet kinetics may show some different properties worth studying. Droplet impact experiments have been used to investigate fluid dynamics and material surface properties, especially for superhydrophobic surfaces. However, researches are mainly focused on the dynamic process of droplet impinging on the wall and the analysis of droplet shape and pressure change during impingement, and the droplet impinges on the surface perpendicularly, while on the droplet colliding with the surface with different microstructural surface Changes in the infiltration state and its influence on the surface hydrophobicity have not been reported [8-10]. In this paper, the droplet and the dynamic impact two ways to start the droplet in the micropatterned surface of the movement process of numerical simulation of the droplet steady-state changes in shape and steady-state contact angle changes, the micro-patterned The influence of structural parameters on static contact angle, dynamic spreading of droplets, shrinkage and rebound is intended to provide technical support for the design of the associated high-speed spray equipment.

\section{Methodology}

\subsection{Model setup}
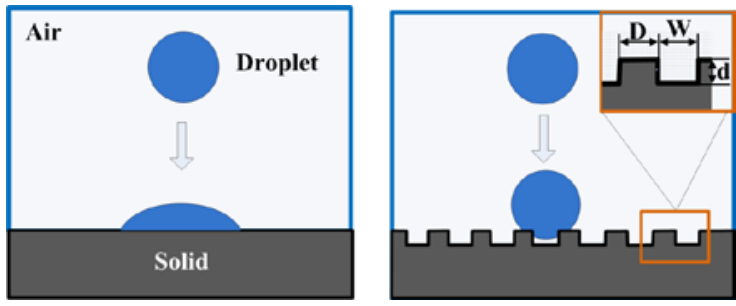

Figure 1 Schematic of the physical model.

Figure 1 shows the schematic of the physical model. In the simulation, the liquid phase is set as water, and its specific physical parameters are: density $\rho=1000 \mathrm{~kg}$ / $\mathrm{m} 3$, viscosity $\mu=1 \times 10-3 \mathrm{~Pa} \cdot \mathrm{s}$, and surface tension $\sigma=7.35 \times 10-2 \mathrm{~N} / \mathrm{m}$. Set the acceleration of gravity $\mathrm{g}=9.8 \mathrm{~m} 2 / \mathrm{s}$, and its direction is vertical down, the initial shape of the droplet spherical diameter is $2 \mathrm{~mm}$. A two-dimensional model was created using preprocessing software workbench. The simulated area was an $8 \times$ $10 \mathrm{~mm}$ symmetric 2-dimensional area. The patterned dimensions are: $\mathrm{W}=60 \mu \mathrm{m}, \mathrm{D}$ $=60 \mu \mathrm{m}, \mathrm{d}=20 \mu \mathrm{m}$. In the process of simulation, the shape of gas-liquid free interface changes obviously. The uniform grid of quadrilateral is used to divide the quadrilateral grid into a total of 40,000 to 45,000 quadrilateral grids. ANSYS12 FLUENT was used for numerical calculation and post-processing. The solver used in the numerical model was pressure based implicit algorithm, multiphase flow model was VOF model, and pressure-velocity coupling method was Fractional Stop 
Academic Journal of Engineering and Technology Science

ISSN 2616-5767 Vol. 1, Issue 1: 49-55, DOI: 10.25236/AJETS.020006

method. The wall of the lower boundary of the simulation area is set as the wall with hydrophilic property, the contact angle of the wall is set to be $70^{\circ}$, and the rest of the boundaries are set as the pressure inlet boundary and the pressure is zero. The boundary of the wall is free of slip boundary conditions, is the velocity components are all zero.

\subsection{Boundary conditions and parameters}

Firstly, the effect of surface patterned parameters on the hydrophobic properties of the surface is studied by simulating the droplet and the dynamic impact. The geometrical parameters are shown in Table 1. Droplet simulation process: Firstly, control the droplet height of $1.5 \mathrm{~mm}$, the initial velocity of 0 , making the solid-liquid contact critical speed approaching 0. Dynamic impact process simulation: Based on the above simulation process, the landing speed of the droplet is introduced, and the critical velocity of the impact is controlled to be $0.5,1,1.5$ and $2 \mathrm{~m} / \mathrm{s}$ respectively to observe the dynamic process of the droplet and to stabilize Changes in state contact angle. When the droplets are stable on the patterned surface, the steady-state contact angle is calculated.

\section{Results and discussion}

\subsection{Overall structure design of the intelligent workshop product}

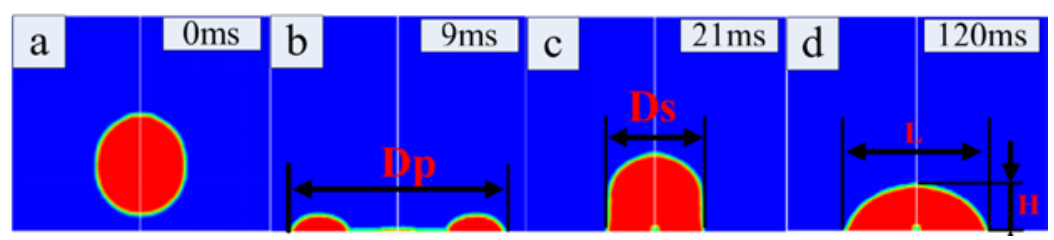

Figure 2 Process of droplet impact on the surface.

When the droplet has a certain velocity impacting the surface of the material, the dynamic process of the droplet generally goes through four stages, as shown in Figure 2. Droplets droplet at a certain height (Figure 2a). Droplets with larger kinetic energy gradually spread due to inertia when they contact the surface, until they reach the maximum spreading diameter (Figure 2b), begin to retract under inertia and reach a minimum Shrink the diameter (Figure 2c) and then circulate the above process and finally stabilize (Figure 2d). Shown in Figure 2 for the static contact angle calculation diagram, in which independent variable $\mathrm{H}$ can be calculated by the number of grid calibration (single grid side length of $0.05 \mathrm{~mm}$ ); argument $\mathrm{L}$ by observing the contact line contains the number of dimples Calibration. The contact angle is then calculated according to the equation: $\theta=2 \tan ^{-}$ ${ }^{1}(2 \mathrm{H} / \mathrm{L})$. 
In the simulation, assuming that the solid wall is not modified or patterned with low surface energy, the solid contact angle of the solid wall in the contact area of the droplet is $70^{\circ}$, at which point there is a great adhesion between the droplet and the surface. Figure 3 shows the simulated contact angle $\theta$ and theoretical contact angle $\theta \mathrm{c}$ versus $\mathrm{A}$, where $\mathrm{A}$ represents the dimples dimensionless surface density.

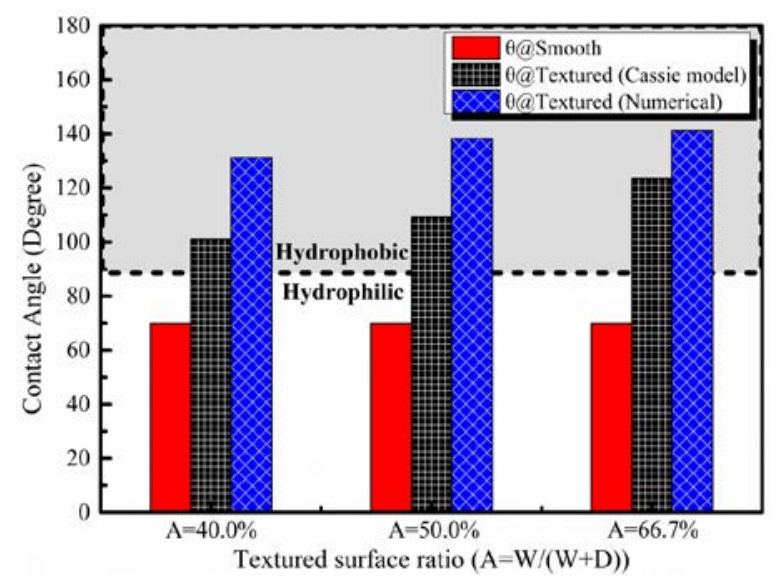

Figure 3 Static contact angle of the theoretical and numerical simulation.

The contact angle of the original solid, which has not been modified with low surface energy or patterned, is assumed as $70^{\circ}$ (as most of metals alloy). Figure 3 shows the simulated contact angle $\theta$ and theoretical contact angle $\theta \mathrm{c}$ versus A. A represents the dimples dimensionless surface density. As is shown in Figure 3, the calculated value of $\theta$ increases with the increase of $A$ and is positively correlated with $\mathrm{A}$, and the simulation results show that the calculated value of contact angle keeps the same trend with the theoretical value. The contact angle $\theta$ value changes with respect to the dimple size under different micro-patterned surface densities. As shown in the figure, the static contact angle of the droplets on the wall surface with the dimple density of $40 \%, 50 \%$ and $66.7 \%$ and the dimple diameter of $60 \mu \mathrm{m}$ is $141^{\circ}$. 


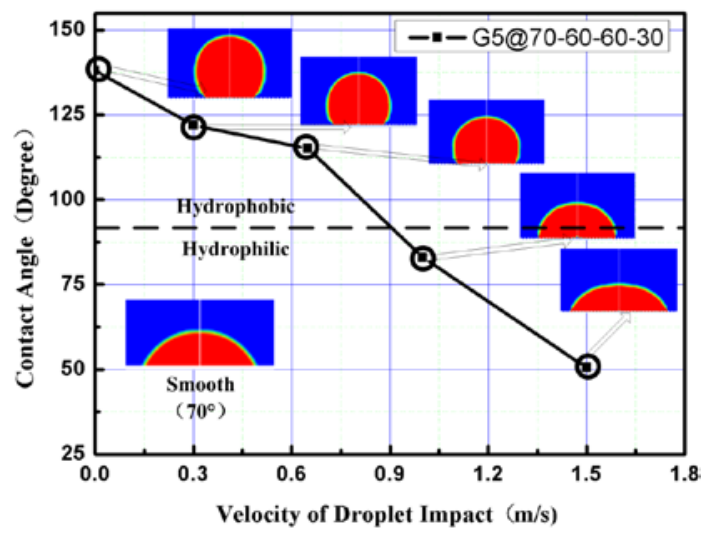

Figure 4 Steady-state contact angle of droplet with landing speed.

Figure 4 shows the trend of the final steady-state contact angle with the landing speed of the patterned surface (dimple spacing of $60 \mu \mathrm{m}$, dimple diameter of $60 \mu \mathrm{m}$, depth of $30 \mu \mathrm{m})$. From the overall trend, the dynamic contact angle of droplets gradually decreases from high hydrophobicity $\left(138^{\circ}\right)$ to hydrophilicity $\left(50.5^{\circ}\right)$, even lower than the inherent contact angle of smooth surface, as droplet landing speed increases. It can be clearly seen from the figure that when the velocity is less than $0.6 \mathrm{~m} / \mathrm{s}$, the dynamic contact angle of droplets slightly, but the hydrophobicity remains high $\left(\theta=118.1^{\circ}\right)$. However, when the landing speed continues increasing, the dynamic contact angle decreased obviously and became the hydrophilic surface $\left(83^{\circ}\right)$. Therefore, it is inferred that there has a critical velocity during the transformation of the wetting state from the Cassie state to the Wenzel state with the change of the velocity. When the collision velocity is greater than critical velocity, the dynamic contact angle of the surface droplet, and then hydrophobic property is reduced sharply.

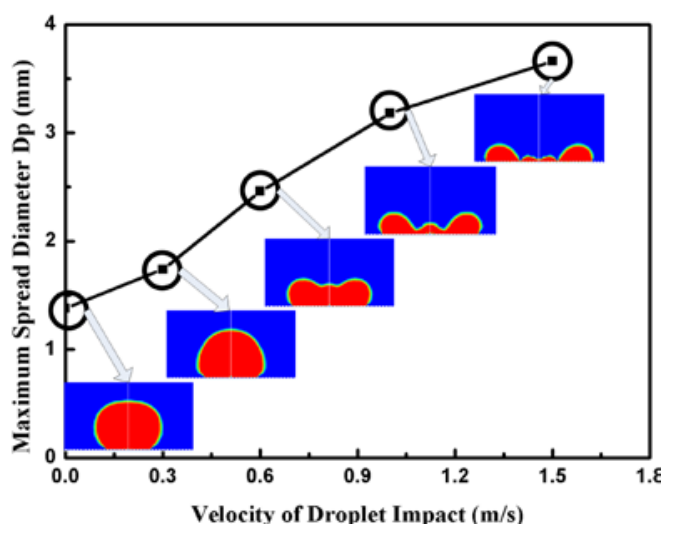

Figure 5 Maximum droplet diameter Dp vs. landing speed. 
Academic Journal of Engineering and Technology Science

ISSN 2616-5767 Vol. 1, Issue 1: 49-55, DOI: 10.25236/AJETS.020006

From Figure 5, the maximum spreading diameter Dp of the droplet gradually increases with the increase of the landing speed of the droplet, which is opposite to the change of the contact angle. This is mainly attributed to that when the landing speed is increased, the kinetic energy of the droplets also increases correspondingly, so the maximum spreading diameter that the droplet can reach is gradually increased, while the increased spreading diameter means that the solid-liquid will continue to increase. Since this simulation formulates the intrinsic contact of the patterned surface Angle of $70^{\circ}$, with hydrophilic properties, the surface of the droplet adhesion as the leading role, and this adhesion will increase with the contact area increases gradually, especially when the droplets shrink due to inertial action, Seriously hinder the droplet movement, eventually leading to the droplet contact angle will decline with increasing speed.

\section{Conclusion}

By changing the landing speed, the droplet dynamic properties are examined in the impacting on the hydrophilic patterned surface status. The conclusions are as follows:

(1) The numerical results demonstrate that the static contact angle is in great concurrence with the Cassie-Baxter equation when the landing speed $\mathrm{V}$ approaches 0 , however the outcome shows that the distinction among $\theta$ and $\theta \mathrm{c}$ is around $30^{\circ}$.

(2) When the droplet landing speed is step by step expanded, the dynamic contact angle of droplets steadily changes from superhydrophobicity $\left(138^{\circ}\right)$ to high hydrophibicity $\left(50.5^{\circ}\right)$. The transition of wetting state is basically caused by the change of wetting status. It is additionally discovered that there is a basic speed in the progress of the wetting state from Cassie state to Wenzel state as the speed changes. At the point when the landing speed is more noteworthy than the basic speed, the dynamic contact angle of the surface drops significantly.

(3) The most extreme spreading diameter and the landing speed are straightly related. The more noteworthy the speed, the more prominent the most extreme spreading breadth. Besides, the bigger the adhesive force of the surface, the littler the contact angle.

\section{Acknowledgements}

This work was financially supported by the National Natural Science Foundation of China via grant number 51505194, the Natural Science Foundation of Jiangsu Province via grant number BK20150517 and the Senior Talent Start-up Foundation of Jiangsu University via grant number 15JDG033. 


\section{References}

[1] S. Xie and Y. Wang (2014). Construction of tree network with limited delivery latency in homogeneous wireless sensor networks. Wireless Personal Communications, vol.78, no.1, p.231-246.

[2] S.-F. Ou, K.-K. Wang and Y.-C. Hsu (2017). Superhydrophobic NiTi shape memory alloy surfaces fabricated by anodization and surface mechanical attrition treatment, Applied Surface Science, vol.425, p.594-602.

[3] S. Cui, S. Lu, W. Xu, B. An and B. Wu (2017). Fabrication of robust gold superhydrophobic surface on iron substrate with properties of corrosion resistance, self-cleaning and mechanical durability", Journal of Alloys and Compounds, vol.728, p.271-281.

[4] E. Shafrin and W. Zisman (1967). Critical surface tension for spreading on a liquid substrate, Journal of Physical Chemistry, vol.71, no.5, p.1309-1316

[5] B. Yilbas, A. Al-Sharafi, H. Ali and N. Al-Aqeeli (2017). Dynamics of a water droplet on a hydrophobic inclined surface: influence of droplet size and surface inclination angle on droplet rolling, RSC Advances, vol.7, no.77, p.48806-48818.

[6] Y. Zhang, H. Xia, E. Kim and H. Sun (2012). Recent developments in superhydrophobic surfaces with unique structural and functional properties, Soft Matter, vol. 8, no.44, p.11217-11231.

[7] S. Nguyen, H. Webb, P. Mahon, R. Crawford and E. Ivanova (2014). Natural insect and plant micro-/nanostructsured surfaces: an excellent selection of valuable templates with superhydrophobic and self-cleaning properties, Molecules, vol. 19, no.9, p.13614-30.

[8] J. Behrndt and H. Kreusler (2013). Spray impingement modeling: Evaluation of the dissipative energy loss and influence of an enhanced near-wall treatment, Fuel Processing Technology, vol. 107, no.3, and p.71-80.

[9] A. Moqaddam, S. Chikatamarla and I. Karlin (2017). Drops bouncing off macrotextured superhydrophobic surfaces, Journal of Fluid Mechanics, vol. 824, p.866-885.

[10] J. Quek, C. Magee and H. Low (2017). Physical texturing for superhydrophobic polymeric surfaces: a design perspective, Langmuir, vol. 33, no.27, p6902-6915

[11] H. Almohammadi and A. Amirfazli (2017). Asymmetric Spreading of a Drop upon Impact onto a Surface”, Langmuir, vol. 33, no. 23, p.5957-5964.

Published by Francis Academic Press, UK 